\title{
Flexible Unidirectional PTD-Symmetric Waveguide
}

\author{
Iram Nadeem, Enrica Martini, Alberto Toccafondi, and Stefano Maci
}

\begin{abstract}
The transmission and reflection characteristics of a bent square transverse electromagnetic waveguide constituted by two opposite perfect electric conductor and perfect magnetic conductor walls are analyzed. This waveguide exhibits a parity, timereversal, duality (PTD) symmetry with respect to both the diagonal axes. It is found that this property is maintained even when the structure is bent in a plane orthogonal to one of the two PTD symmetry axes (PTDbend). As a consequence, the transverse electromagnetic mode propagation is protected against backscattering by this class of discontinuities. The preservation of the PTD symmetry in presence of a geometric flexibility of the bend is also analyzed, thus introducing a new class of bendable waveguides that are largely immune to backscattering by bend discontinuities.
\end{abstract}

\section{Introduction}

The recent understanding of topological states of matter has yielded new opportunities for the manipulation of electromagnetic waves [1,2]. In particular, recent studies provided an easy way to classify bandgap materials by evaluating their topological invariants [3]. Materials with null invariant are trivial, and they behave in the same way as ordinary materials. However, if the invariant is different from zero, the geometric phase effects become relevant, with significant interesting physical consequences [4]. These consequences are related to the presence of a highly robust transport mode (existing within the bandgap) at the boundary between nontrivial and significant materials. These edge modes [5] are the consequence of the quantization of conductivity in quantum Hall effects [6]. Thus, the novel aspect of topological states is that they deal with edge modes, named topological edge modes, that can be unidirectional and thereby protected against backscattering [7]. This allows for the conception of guiding structures that are free from undesired effects of reflections due to disorder, imperfections, obstacles, or deformations of the propagation path. Nevertheless, the protection of topological edge modes normally requires nonreciprocal materials [8]. Recently, it was shown that a similar effect may also be obtained using reciprocal materials $[9,10]$. Topological protected edge modes may therefore occur in time-reversal-invariant structures. The edge modes in this case are bidirectional but largely immune to backscattering [11]. In this case, the key property that guarantees that the propagating mode is immune to

Manuscript received 28 December 2021.

Iram Nadeem, Enrica Martini, Alberto Toccafondi, and Stefano Maci are with the University of Siena, via Roma 56, 53100, Siena, Italy; e-mail: iram.nadeem@student.unisi.it, martini@dii.unisi.it, albertot@dii.unisi.it,macis@dii.unisi.it. reflections is a duality link between the constitutive parameters of the relevant materials $[12,13]$. In [11] it was shown for the first time that a scattering matrix with $S_{11}=\cdots=S_{N N}=0$ characterizes a large family of bidirectional (not necessarily reciprocal) $N$-port networks that are invariant under the combined action of the parity (P), time-reversal (reciprocity; T), and duality (D) operators. As a result, a microwave network with parity, time-reversal, duality (PTD) invariance is always matched at all ports. Unlike topology, PTD invariance (or PTD symmetry) is a single-frequency condition rather than a global spectral property. Also, it requires only reciprocal materials, which simplifies fabrication. Topological insulators described in [14] are examples of PTDsymmetric systems with an $\Omega$-type bianisotropic coupling. Furthermore, the waveguide structures formed by a junction between complementary impedance surfaces $[15,16]$ are also PTD-symmetric waveguides. An array of open-ended square PTD-symmetric waveguides was investigated in $[17,18]$. This array with ideal boundary conditions fulfills PTD symmetry, and it allows wideangle beam scanning with good matching performance. Finally, in $[19,20]$ a PTD-symmetric closed transversedomain guiding structure in a parallel-plate waveguide was introduced and analyzed. An exact solution for the protected transverse electromagnetic (TEM) edge mode supported by this structure was derived in [19].

In this article, we analyze the properties of a PTDsymmetric closed square waveguide composed of two perfect electric conductor (PEC) and two perfect magnetic conductor (PMC) walls. This structure supports a TEM mode which has the structure of a plane wave [21], and was studied a long time ago. However, only recently has attention been given in the literature to its symmetry-protected transport property [13]. As a matter of fact, this TEM waveguide satisfies PTD symmetry for inversion of one diagonal axis. As a consequence, the waveguide may be bent in a plane that maintains one diagonal axis invariant without losing the protection with respect to backscattering.

This article is organized as follows. Section 2 presents the geometry and some considerations on an ideal PTD-symmetric square TEM waveguide open into free space. Section 3 shows the properties of two different examples of right-angle bent TEM waveguides, one not respecting PTD symmetry. Section 4 analyzes transmission and reflection characteristics of a bendable waveguide, and shows its performance when the bend angle is decreased from $90^{\circ}$ up to an almost vanishing angle. Conclusions are finally drawn in Section 5.

\section{PTD-Symmetric Waveguide}

The geometric configuration of a square TEM waveguide, PTD symmetric with respect to both the 


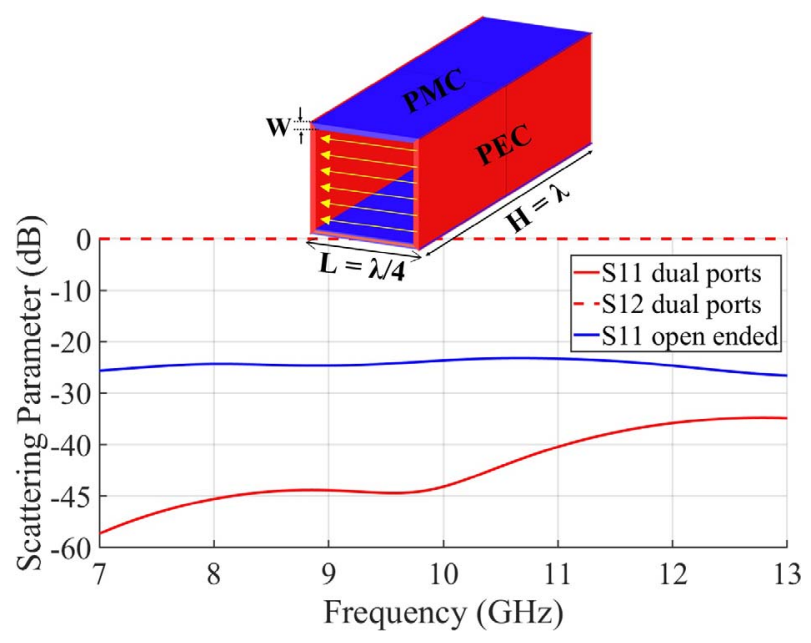

Figure 1. Amplitude of the reflection and transmission coefficients versus frequency for a straight section of PTD-symmetric square waveguide, calculated with HFSS.

diagonal axes, is shown in the inset of Figure 1. This waveguide can be realized by using printed metasurfaces on two opposite walls [21] to mimic the PMC boundary conditions. It is well known that the ideal structure supports a TEM mode, namely a mode without cutoff [21]. However, in its practical implementation with metasurfaces, the supported mode does not have a zerocutoff frequency, and it exists only in a certain bandwidth around the frequency where the metasurface exhibits an equivalent PMC behavior.

A basic PTD-symmetric waveguide straight section has been designed and analyzed using HFSS HFSSv2021 commercial software. The length of each wall is $L=\lambda / 4$ at $f_{0}=7 \mathrm{GHz}, W=0.5 \mathrm{~mm}$, and the overall length of the waveguide along the propagation axis is $H=\lambda$. Two cases have been analyzed. In the first case, two ideal ports (with the configuration of the TEM mode) have been set at the two end points, and the transmission (red dashed line) and reflection (red continuous line) coefficients have been calculated. The simulated reflection coefficient at the waveguide port is below $-35 \mathrm{~dB}$, and the transmission coefficient is close to $0 \mathrm{~dB}$. This first example has no practical relevance except that it calibrates the level of numerical noise in modeling the structure with HFSS ports. In the second example, the waveguide is open-ended into free space, and only one ideal port is applied at one side. The PEC and PMC boundary conditions have been applied in HFSS even to the external sides of the walls. It is seen that the reflection coefficient (blue line) is quite low for all the frequencies considered. The reason for this good matching is that free space is a particular type of PTD-symmetric medium, and therefore the discontinuity introduced by the termination is PTD-symmetry compliant.

\section{Reflection Properties of $90^{\circ}$ Bend Discontinuities}

Let us consider first the waveguide cross section in Figure 2, where the $z$-axis is the PTD symmetry axis.

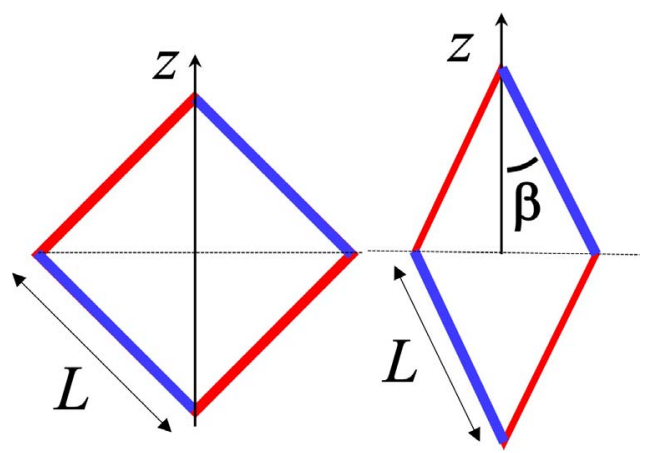

Figure 2. Cross-section geometry of the square and rhombic waveguides with the same side length. PMC walls are represented in blue and PEC walls in red.

This means that changing $z$ to $-z$ makes the boundary conditions dual. The cross section can be square (left) or rhombic (right) with the PTD symmetry property maintained. In order to assess the impact of the PTD theory on the reflection properties of a bend, we have considered two types of right-angle bend for a PTDsymmetric TEM waveguide. For simplicity, we will consider first the square cross section. In the first configuration, the bend is made in the plane orthogonal to the parity axis - i.e., connecting two straight sections of square cross section with the propagation axis in the $x y$-plane. This geometry fulfills the PTD symmetry property overall, and therefore we denote it a "PTDbend." In the second configuration, the bend is made around one of the two PEC walls. The two bent sections now share the two planes on which the PMC walls lie. We denote this a "non-PTD-bend."

A PTD-bend in a square TEM waveguide is shown in the inset of Figure 3; the figure also shows the reflection and transmission coefficients. The reflection coefficient is below $-50 \mathrm{~dB}$ in the frequency range between 7 and $13 \mathrm{GHz}$. This means that the structure presents complete transmission, with no reflection inside the waveguide at the bend discontinuity. A snapshot of the electric-field distribution at $11 \mathrm{GHz}$ on the plane shared by the two sections is shown in Figure 4. The electric field excited at one port clearly follows

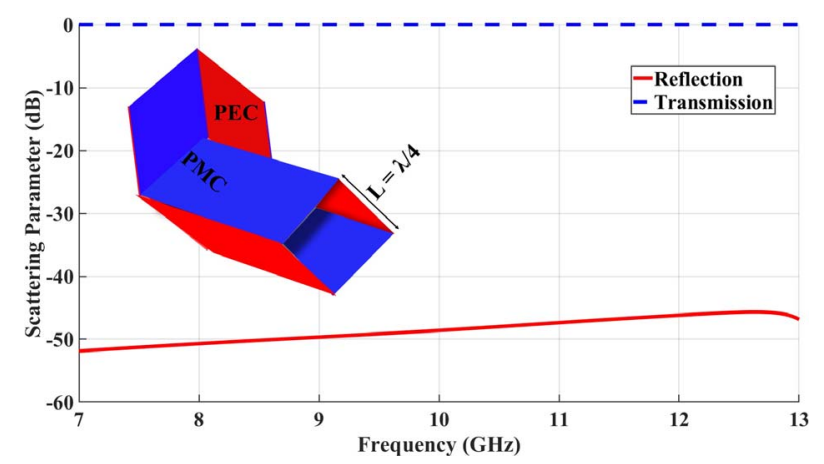

Figure 3. Simulated scattering parameters of PTD-symmetric waveguide with PTD-bend. 


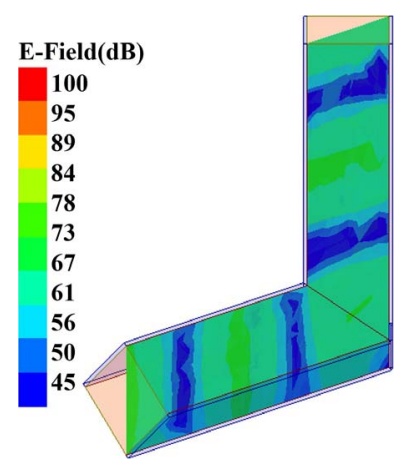

Figure 4. Snapshot of electric field inside the PTD-symmetric waveguide with PTD-bend at $11 \mathrm{GHz}$.

the path between the PMC and PEC walls, and the distortion due to the bend does not give rise to a reflected field at the cross-section discontinuity.

A non-PTD-bend in a square TEM waveguide is shown in the inset of Figure 5. The bend in this case breaks PTD symmetry, and this affects the scattering parameters (also reported in the figure). The reflection coefficient is around $-5 \mathrm{~dB}$ at $f=7 \mathrm{GHz}$ and gradually increases up to almost $0 \mathrm{~dB}$ at $f=13 \mathrm{GHz}$. As a consequence, in the same frequency range the transmission coefficient decreases from $-1 \mathrm{~dB}$ to $-40 \mathrm{~dB}$. This clearly shows that the field is mostly reflected back at the bend discontinuity. A snapshot of the electricfield distribution at $f=11 \mathrm{GHz}$ on the plane orthogonal to the PEC walls is shown in Figure 6. The field excited at one port does not reach the other port, due to the strong reflection at the right-angle bend.

\section{Flexible PTD-Symmetric Waveguide}

A PTD-symmetric bent TEM waveguide, with an arbitrary bend angle, exhibits an interesting geometric (and mechanical) property: starting with an initial bent configuration with a square cross section, the bending angle can be increased continuously, changing the cross section from a square to a rhombus, without breaking the PTD symmetry of the resulting waveguide. This realizes a flexible PTD-symmetric waveguide. Let us

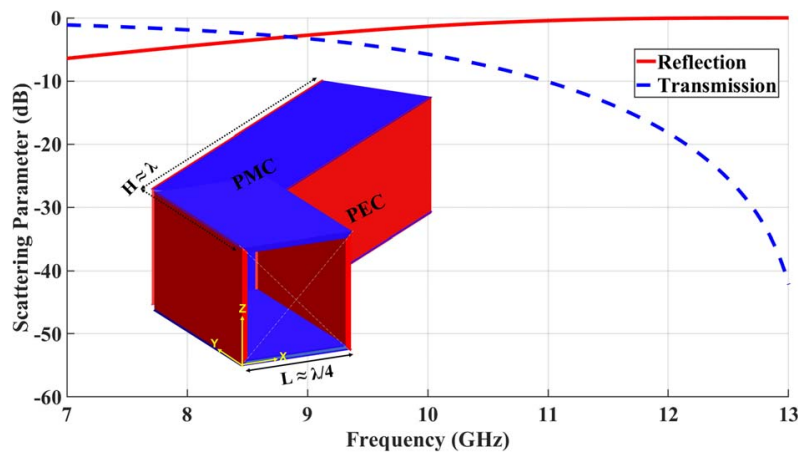

Figure 5. Simulated scattering parameters of PTD-symmetric waveguide with non-PTD-bend.

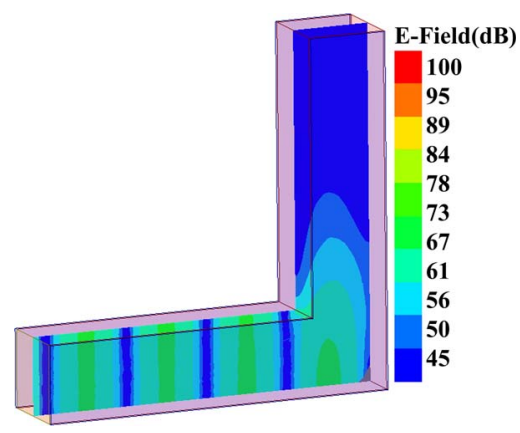

Figure 6. Snapshot of electric field inside the PTD-symmetric waveguide with non-PTD-bend at $11 \mathrm{GHz}$.

consider the right-angle PTD-symmetric bent waveguide shown on the left of Figure 7, which is the same as that in Figure 4. Let us denote with $\beta$ the half-angle between PEC and PMC walls across the PTD axis (zaxis). Moreover, let us denote with $\alpha$ the half-angle between the symmetry axes of the two straight waveguide sections - i.e., the $x$-axis and $\eta$-axis in Figure 7. The flexibility of the PTD-symmetric waveguide is implemented by varying $\beta$ between $45^{\circ}$ and $0^{\circ}$. The following relationship holds between the distance $\left|P_{1}-P_{2}\right|$ between the two points $P_{1}$ and $P_{2}$ and the two angles $\alpha$ and $\beta$ :

$$
\begin{gathered}
P_{1}=(L \sin \beta \cot \alpha, 0, L \sin \beta) \\
P_{2}=(0, L \cos \beta, 0) \\
\left|P_{1}-P_{2}\right|=L \sqrt{1+\sin ^{2} \beta \cot ^{2} \alpha}
\end{gathered}
$$

Since the distance $\left|P_{1}-P_{2}\right|$ has to remain constant during the folding operation in order to ensure that the structure is mechanically realizable, the quantity $\sin \beta \cot \alpha$ must also remain constant. Its value is found by imposing one desired angle combination; for example, $\alpha=\beta=\pi / 4$ (square waveguide cross section for a $90^{\circ}$ bend) gives $\left|P_{1}-P_{2}\right|=L \sqrt{3 / 2}$, which means $\sin \beta \cot \alpha=L / \sqrt{2}$.

Figure 8 shows some examples of geometry variations for different value of $\beta$. It is worth noting that starting with a square cross section for a $90^{\circ}$ bend,

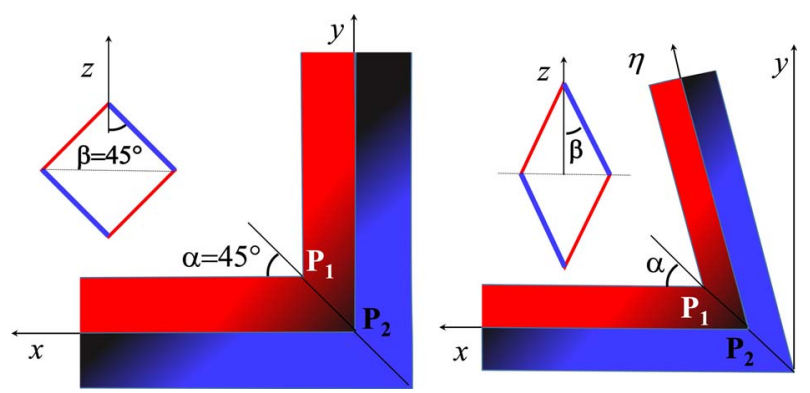

Figure 7. Flexible PTD-symmetric waveguide and relevant geometric parameters. 

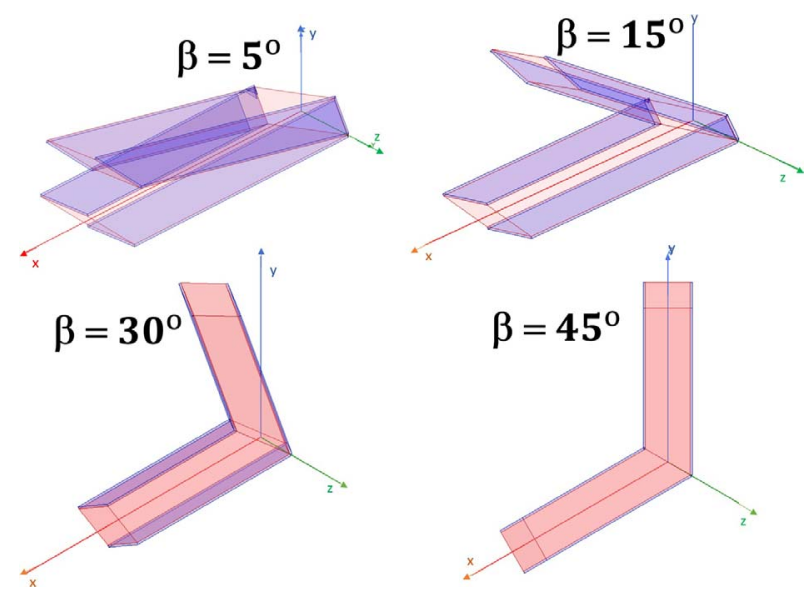

Figure 8. Geometry of PTD-symmetric waveguide bend for different values of $\beta$.

the cross section becomes rhombic in the folding operation.

Figure 9 gives the simulated reflection and transmission parameters for different values of $\beta$. For all the values considered, the transmission coefficient is close to $0 \mathrm{~dB}$ for a considerably large frequency band. The worst performances are obtained for $\beta=5^{\circ}$, when the aperture of the waveguide based on PEC and PMC walls is very narrow. For higher values of $\beta$ the reflection is less than $-38 \mathrm{~dB}$ over the entire band between 7 and $13 \mathrm{GHz}$. The characteristic impedance of the TEM mode is approximately $377 \Omega$ for all the $\beta$ angles.

A snapshot of the electric field in the $x y$-plane of the PTD-symmetric bent waveguide is presented in Figure 10. The field propagates with very low backscattering.

\section{Conclusion}

In this article, the properties of a bent PTDsymmetric square waveguide constituted by PEC and PMC walls were investigated. This waveguide supports unimodal TEM mode propagation protected against backscattering from deformations and defects that preserve PTD symmetry. This property is also preserved

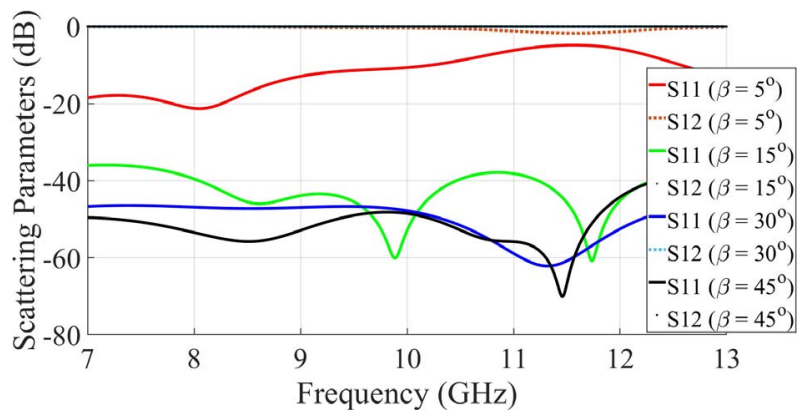

Figure 9. Simulated scattering parameters of the PTD-symmetric waveguide bend for different values of $\beta$.

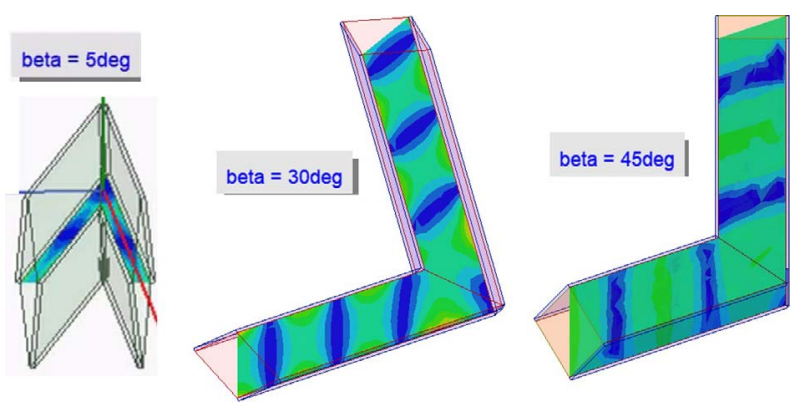

Figure 10. Snapshot of the simulated electric field of a PTDsymmetric waveguide bend for different vales of $\beta$.

when the bent waveguide is folded, thus yielding a flexible waveguide. The characteristic impedance of the TEM mode coincides with the wave impedance of free space, for all the folded configurations.

\section{References}

1. J. Joannopoulos, SG. Johnson SG, JN. Winn, RD. Meade, "Photonic Crystals: Molding the Flow of Light." Princeton, NJ: Princeton Univ.Press, 2008

2. F. D. M. Haldane and S. Raghu, "Possible Realization of Directional Optical Waveguides in Photonic Crystals with Broken Time-Reversal Symmetry," Physical Review Letters, 100, 1, January 2008, p. 013904.

3. M. Z. Hasan and C. L. Kane, "Colloquium: Topological Insulators," Reviews of Modern Physics, 82, 4, OctoberDecember 2010, pp. 3045-3067.

4. A. Bansil, H. Lin, and T. Das, "Colloquium: Topological Band Theory," Reviews of Modern Physics, 88, 2, AprilJune 2016, p. 021004.

5. X.-L. Qi and S.-C. Zhang, "Topological Insulators and Superconductors," Reviews of Modern Physics, 83, 4, October-December 2011, pp. 1057-1110.

6. F. D. M. Haldane, "Model for a Quantum Hall Effect Without Landau Levels: Condensed-Matter Realization of the 'Parity Anomaly," Physical Review Letters, 61, 18, October 1988, pp. 2015-2018.

7. Z. Wang, Y. Chong, J. D. Joannopoulos, and M. Soljačić, "Observation of Unidirectional Backscattering-Immune Topological Electromagnetic States," Nature, 461, 7265, October 2009, pp. 772-775.

8. H. Lira, Z. Yu, S. Fan, and M. Lipson, "Electrically Driven Nonreciprocity Induced by Interband Photonic Transition on a Silicon Chip," Physical Review Letters, 109, 3, July 2012, p. 033901.

9. M. C. Rechtsman, J. M. Zeuner, Y. Plotnik, Y. Lumer, D. Podolsky, et al., "Photonic Floquet Topological Insulators," Nature, 496, 7444, April 2013, pp. 196-200.

10. W. Gao, M. Lawrence, B. Yang, F. Liu, F. Fang, et al, "Topological Photonic Phase in Chiral Hyperbolic Metamaterials," Physical Review Letters, 114, 3, January 2015, p. 037402.

11. M. G. Silveirinha, "P · T · D Symmetry-Protected Scattering Anomaly in Optics," Physical Review B, 95, 3, January 2017, p. 035153.

12. F. Liu and J. Li, "Gauge Field Optics With Anisotropic Media," Physical Review Letters, 114, 10, March 2015, p. 103902.

13. W.-J. Chen, Z.-Q. Zhang, J.-W. Dong, and C. T. Chan, "Symmetry-Protected Transport in a Pseudospin-Polar- 
ized Waveguide," Nature Communications, 6, 1, September 2015 , p. 8183 .

14. A. B. Khanikaev, S. H. Mousavi, W.-K. Tse, M. Kargarian, A. H. MacDonald, et al, "Photonic Topological Insulators," Nature Materials, 12, 3, March 2013, pp. 233-239.

15. D. J. Bisharat and D. F. Sievenpiper, "Guiding Waves Along an Infinitesimal Line Between Impedance Surfaces," Physical Review Letters, 119, 10, September 2017, p. 106802.

16. Z. Xu, J. Chang, S. Fang, Q. Zhang, R. J. Davis, et al, "Line Waves Existing at Junctions of Dual-Impedance Metasurfaces," ACS Photonics, 8, 8, June 2021, pp. 22852293.

17. I. Nadeem, V. Valentina, E. Martini, A. Toccafondi, and S. Maci, "Active Impedance of PTD-Symmetric Checkerboard Type Open Ended Waveguide Array," In Proceedings of the 15th European Conference on Antenna and Propagation, Virtual Conference, 2021, Location, Germany, 6 March 20-26, 2021, pp. PPP.

18. I. Nadeem, V. Valentina, E. Martini, A. Toccafondi, and S. Maci, "PTD-Symmetric Square-Waveguide Array
Based on Complementary Boundary Conditions," In Proceedings of the 15th International Congress on Artificial Materials for Novel Wave Phenomena-Metamaterials 2021, Location New York, USA, September 20 23, 2021 pp. $215-217$

19. E. Martini, M. G. Silveirinha, and S. Maci, "Exact Solution for the Protected TEM Edge Mode in a PTDSymmetric Parallel-Plate Waveguide," IEEE Transactions on Antennas and Propagation, 67, 2, February 2019, pp. 1035-1044.

20. E. Martini, M. G. Silveirinha, and S. Maci, "Transformation Method for PTD Symmetric Edge Waveguide," 2019 13th European Conference on Antennas and Propagation (EuCAP). Krakow, Poland, March 31-April 5, 2019, pp. 1-3.

21. M. N. M. Kehn and P.-S. Kildal, "The N-Guide: A Novel Miniaturized Hard Quasi-TEM Waveguide," IEEE Antennas and Propagation Society International Symposium. Digest. Held in conjunction with: USNC/CNC/URSI North American Radio Sci. Meeting, Columbus, OH, USA, June 22-27, 2003, 2. pp. 1111-1114. 